PALABRAS CLAVE

Sociedad civil

Instituciones sociales

Integración social

Política social

Gobernabilidad

Estudios de casos

Chile

Aldo Mascareño

Doctor en Sociología,

Universidad de Bielefeld

Director del Departamento de Sociología

de la Universidad Alberto Hurtado

- amascaren@uahurtado.cl
REVISTA CEPAL 101 AGOSTO 2010

\section{Coordinación social} mediante políticas públicas: el caso chileno

\author{
Aldo Mascareño
}

$\mathrm{L}$

a complejidad social supone la interrelación de distintos actores y sistemas con marcada autonomía para definir intereses y procedimientos de operación propios. En este contexto se desarrollan modelos de coordinación social en que se combina la autonomía de actores y sistemas con la coherencia en torno de objetivos. Sobre la base de ejemplos del caso chileno, en el artículo se revisan: i) los modelos de policy networks o redes de políticas públicas (sistema de concesiones); ii) los sistemas de deliberación (comisiones asesoras presidenciales) y iii) los sistemas de derecho reflexivo (arbitraje comercial internacional). Se concluye que la alta reflexividad de estos modelos permite, aun con limitaciones, la combinación de los principios de autonomía y coherencia en la operación de políticas públicas. 


\section{I}

\section{Introducción}

Uno de los procesos más característicos de la sociedad contemporánea es, desde un punto de vista sociológico, su creciente tendencia a una mayor complejidad social (Habermas, 1990; Luhmann, 2007). Esta puede ser descrita en tres dimensiones:

i) En un nivel concreto, supone la proliferación de múltiples organizaciones privadas, públicas, cuasiprivadas o semipúblicas, actores corporativos nacionales y supranacionales, movimientos de protesta de orden transnacional, agrupaciones de representación local o regional y una participación individualizada en diversos espacios sociales diferenciados no siempre consistente y más bien fragmentada.

ii) En una dimensión social, la propensión a una mayor complejidad implica que cada uno de esos campos se organiza en torno de sus propios intereses y procedimientos de operación, por lo que el resultado más probable de tal operación es la colisión de intereses sustantivos y el conflicto entre los procedimientos de cada campo.

iii) Finalmente, en una dimensión temporal, la "complejización" (de ahora en adelante, complejización) social supone que intereses sustantivos y sus procedimientos relacionados establecen prioridades temporales precisas y mecanismos autorregulatorios propios para lograr su cumplimiento, según requerimientos que aseguren la continuidad de cada campo.

En una palabra, la complejización involucra una diferenciación de la sociedad contemporánea en diversos sistemas y actores con creciente autonomía de expectativas y de procedimientos operativos.

América Latina y Chile en particular no han estado ajenos a este proceso de complejización. En Chile, al menos en las últimas tres décadas, a las clásicas organizaciones sindicales y actores populares se ha sumado una serie de nuevas agrupaciones sobre la base de intereses diversos: grupos juveniles, de tercera edad, feministas, homosexuales, migrantes, ambientalistas, agrupaciones comunitarias territoriales, de derechos urbanos, de consumidores, de derechos humanos y ciudadanos, otras de carácter neorreligioso, comunidades artísticas, y movimientos indígenas y estudiantiles

$\square$ Este artículo forma parte del Proyecto del Fondo Nacional de Desarrollo Científico y Tecnológico (FONDECYT) № 1095186. de distinto tipo. A ello se agregan la diversificación de entidades públicas para atender a estos actores (nuevos ministerios, subsecretarías, superintendencias, agencias regulatorias), mecanismos como mesas de negociación, comités de expertos, comisiones de estudio, de ética, así como la proliferación de organizaciones del denominado tercer sector, organizaciones no gubernamentales (ONG), organizaciones económicas nacionales y transnacionales y variados agentes privados en diversos campos sociales transnacionales (Domingues, 2008).

Toda esta diversificación concreta de sistemas, organizaciones y actores implica la emergencia de intereses sustantivamente contradictorios entre sí, que ganan en autonomía al establecer sus propios procedimientos de funcionamiento y agendas temporales para la consecución de sus expectativas. Frente a esto, surge la pregunta por la capacidad del Estado, mediante políticas públicas, de absorber y articular demandas diversas, conflictivas entre sí y con expectativas de cumplimiento que no admiten demasiada flexibilidad temporal. El concepto de políticas públicas puede ser entendido como un conjunto de medidas administrativas y jurídicas desplegadas en el marco estatal para el tratamiento de problemas sociales y la orientación de actores a determinadas conductas (Kraft y Furlong, 2009).

Durante el siglo XX, las políticas públicas en Chile siguieron dos modelos opuestos en períodos históricos distintos: uno de tipo Estado-céntrico caracterizado por una considerable intervención estatal de sustrato desarrollista, basada en planificación y orientada a la incorporación de las clases medias y sectores populares (1932-1973), y otro marcado por la retirada del Estado y el énfasis en políticas macroeconómicas (1973-1989) (Arellano, 1985). El período siguiente se caracterizó por un doble movimiento: por una parte, el intento (político) de volver a fórmulas Estado-céntricas previas a 1973 y, por otra, la imposibilidad estructural de hacerlo sobre un modelo notoriamente marcado por reformas neoliberales. Esto es especialmente visible en los sistemas educacional, laboral, de bienes y servicios colectivos, de salud, de pensiones y de seguridad social, todos ellos con elevados niveles de privatización en sus modos de operación (Hecht-Oppenheim, 1993). 
La hipótesis de este artículo es que frente a la imposibilidad de recrear una matriz Estado-céntrica basada en la planificación de políticas públicas y ante la alta autonomía ganada por distintos sistemas y actores, desde 1990 en adelante se comienza a formar en Chile una nueva alternativa para el despliegue de políticas públicas. En ella se combina el papel estatal de orientación social por medio de políticas públicas que procuran la coordinación (y no la intervención Estado-céntrica) de sistemas y actores, con intereses sustantivos propios y procedimientos de operación autónomos.

Para desplegar esta hipótesis se indican, en primer lugar, los elementos centrales para una perspectiva de coordinación social por medio de políticas públicas en contextos complejos (sección II). Luego se revisan en sus fundamentos teóricos y mediante ejemplos respectivos tres modelos de coordinación y su emergencia en el caso chileno: el modelo de policy networks para el caso del sistema de concesiones de obras públicas (sección III), el modelo de sistemas de deliberación para el caso de las comisiones asesoras presidenciales (sección IV) y el modelo de derecho reflexivo o de política de opciones (options policy) para el caso del arbitraje en materias comerciales (sección V). Finaliza el artículo con una aproximación evaluativa de estos modelos a nivel latinoamericano (sección VI).

\section{II}

\section{Elementos centrales para una perspectiva de coordinación social}

Basado en los principios de coherencia y autonomía, Norbert Lechner (1997) ha identificado tres formas básicas de coordinación social: la coordinación política, la coordinación de mercado y la coordinación por redes. Características de la primera son su centralización, jerarquía y orientación exclusivamente pública; propio de la segunda es su carácter descentralizado, horizontal y no intencionado. La tercera forma, que combina comunicación vertical y horizontal, supone dependencia recíproca y se orienta a la articulación de intereses en un tema común mediante una cooperación competitiva. En este trabajo se reserva el concepto de "coordinación" solo para el tercer caso. Ampliar este concepto a los modos de regulación jerárquico-estatales y a los de mercado solo diluye su especificidad y novedad. Lo que Lechner denomina "coordinación política" ha sido regularmente conocido como intervención estatal o control social (Beyme, 1994); y para el caso de la "coordinación de mercado", Hayek (1986) ya habló de "catalaxia" o autorregulación de mercado. El hecho de que los actores de mercado muestren también autonomía en intereses y procedimientos, los constituye en un caso de análisis, no en el paradigma de la coordinación social. La coordinación, en el sentido aquí expuesto, solo surge cuando algún agente — público o privadoprocura regular la dinámica autónoma de actores y sistemas orientando concreta, social y temporalmente sus rendimientos.
En este sentido, el concepto de coordinación propuesto debe distinguirse de otros modelos de relación público-privada:

i) El intervencionismo desarrollista, modelo privilegiado en el contexto latinoamericano durante gran parte del siglo XX, que se caracteriza por el control estatal de la estructura productiva.

ii) El control corporativista, que se distingue por la cooptación y definición estatal de objetivos y orientaciones de acción de agentes privados (visible en contextos populistas).

iii) El institucionalismo normativo, propio de los análisis sobre conflictos de interés y regulaciones de poder.

iv) La autorregulación, característica de la operación de mercado en contextos neoliberales.

La coordinación asume la alta complejidad de las relaciones de actores y sistemas, su autonomía sustantiva y procedimental y la asimetría de intereses entre ellos. Por ello reconoce que esos actores no pueden ser dirigidos de forma autoritativa (como en el modelo intervencionista y corporativista) y que el conflicto de interés puede ser tratado deliberativamente y no solo sometido a transacciones de poder (como en el institucionalismo normativo). Pero a la vez, la coordinación se aparta de la pura autorregulación (propia del orden emergente del mercado) y establece criterios generales para el desarrollo de finalidades específicas en relación con problemas 
de interés público. Coordinación es un balance entre autonomía y coherencia.

Tal balance parece apropiado para enfrentar la dinámica compleja de actores y sistemas. Dos dimensiones se abren a este respecto: una sociológica y otra histórica. El sustrato sociológico de la coordinación social se encuentra en el despliegue del proceso de diferenciación funcional, esto es: "la constitución de diversos sistemas funcionales relativamente autónomos, estructurados en torno a determinadas lógicas internas" (Lechner 1999, p. 49). El sustrato histórico de ella se relaciona con la crisis del modelo de planificación centralizada y del Estado de bienestar europeo, que condujo a una atrofia financiera y a la juridificación de esferas sociales conocida como "euroesclerosis" (Willke, 1995; Peruzzotti, 1999, para el caso latinoamericano). Del proceso de diferenciación funcional emergen sistemas, organizaciones y actores que cuestionan la capacidad del Estado para dirigir sus acciones mediante planificación o intervenciones autoritativas en la definición de sus intereses y procedimientos. De las crisis planificadoras emerge la necesidad de una alternativa que, respetando la autonomía sistémica, sea capaz de orientar su funcionamiento a la realización paralela de expectativas. En el debate en torno de la coordinación social han surgido con claridad tres candidatos que cumplen estas condiciones: las policy networks (Mayntz, 1992 y 1993; Lechner, 1997; Messner, 1999; Scharpf, 2001; Heydebrand, 2003), la orientación contextual por medio de mesas de deliberación (Willke, 1995, 1997), y el modelo de derecho reflexivo o de política de opciones (Teubner, 1993). A modo de síntesis, se puede decir que como estrategias de coordinación social todas ellas:

i) Suponen la capacidad para introducir coherencia en la interrelación de sistemas y actores autónomos, orientándolos a tareas específicas.

ii) Desarrollan una visión común en torno de un área de problemas orientada a la construcción de relaciones de suma positiva.

iii) Promueven un nivel tolerable de autolimitación de la autonomía, sin que ello suponga cuestionar lo central de los intereses de cada participante. iv) Se "operacionalizan" mediante la articulación de procedimientos antes que por principios normativos generalizados.

v) Aspiran a elevar la reflexividad de sistemas y actores por medio de la atención a las consecuencias de sus operaciones autónomas.

La coordinación social mediante políticas públicas conlleva, de este modo, la combinación de dos principios: por una parte, la coherencia que puede aportar una visión panorámica de intereses y procedimientos de distintos sistemas y actores y, por otra, la autonomía de estos para definir esos intereses y autoorganizarse (Scharpf, 1993; Mayntz, 1993; Willke, 1995). La coherencia es aportada por una mirada estatal a los problemas sociales; la autonomía es propia de sistemas y actores en contextos complejos. Con la combinación de ambas se propone la creación de zonas híbridas, en las que las políticas públicas pueden promover y orientar hacia la producción de un bien o servicio, sin que esto signifique intervenir Estado-céntricamente a sistemas y actores autónomos para la generación de aquello. Mientras la coherencia apunta a establecer esfuerzos coordinados entre los involucrados, la autonomía procura que los diversos sistemas y actores comprometidos también puedan obtener rendimientos para sus intereses y operar, en la mayoría de los casos, con sus propios procedimientos. La coordinación por medio de políticas es, en este sentido, una situación de doble contingencia (Luhmann, 2007), es decir, tanto la operación estatal como la de sistemas y actores autónomos mantienen sus expectativas diferenciadas, pero se vinculan concreta, social y temporalmente en un policy issue (cuestiones de política) del que pueden obtener rendimientos diferenciados, pero coordinados. Se trata de una aspiración de suma positiva, antes que un juego de suma cero. Coordinación social es, en tal sentido, una respuesta a la compulsión de integración de políticas Estado-céntricas basadas en planificación, como también un rechazo a la imposibilidad de una "visión común" frente a problemas sociales relevantes para los actores (Willke, 1995, 1997).

En las siguientes secciones se describen separadamente tres modelos de coordinación: policy networks, mesas de deliberación y derecho reflexivo. Su operación se ejemplifica con casos chilenos de la última década. 


\section{III}

\section{El modelo de policy networks y}

\section{su emergencia en Chile}

La pregunta por la interrelación entre instancias autónomas tiene una larga tradición en el análisis organizacional. Se pueden distinguir ahí relaciones diádicas (Hasenfeld, 1972), conjunto de acciones (action sets) (Whetten y Aldrich, 1979) y redes organizacionales (organisational sets) (Granovetter, 1973). Aplicado al problema de las políticas, este enfoque da origen al modelo de policy networks. En principio, este supone la definición de reglas para la realización de compromisos entre agentes públicos y privados, que permitan una distribución de costos y beneficios frente a una decisión común o una solución de problemas; reglas que en cada caso exigen de los participantes limitar de manera voluntaria su libertad de acción; esto puede conducir a un modelo de identidades organizativas, competencias y esferas de interés mutuamente aceptadas (Mayntz, 1992, p. 27 y ss.). De todos modos, no existe una definición única en esta materia. David Marsh y Rod Rhodes (1992) diferencian las policy networks entre comunitarias (pocos participantes, fuertemente integradas, con alta continuidad y orientadas a uno o dos intereses comunes) y temáticas (mayor número de participantes, múltiples intereses y de mayor conflictividad).

Tanja Börzel (1998) ha detallado dos tendencias fundamentales: la alemana, que identifica a las policy networks como una forma de coordinación alternativa a la jerarquía y el mercado, y la anglosajona, que las concibe como un modelo de relación Estado-sociedad en un área determinada. Mark Bevir y David Richards (2009), basados en estudios etnográficos, han agregado una tercera forma, la descentralización de políticas públicas (decentered policy network), basada en las tradiciones y agencia situada de los participantes. Una tendencia similar siguen Pater de Leon y Danielle Varda (2009) con su idea de redes "colaborativas" de políticas públicas (collaborative policy networks), en las que no solo se examina la composición de actores, sino también sus grados de institucionalización y los intercambios discursivos entre ellos.

El enfoque se ha extendido también a los problemas de coordinación en espacios transnacionales como relación de multiniveles de gobernanza - local, regional, nacional, supranacional, global (Scharpf, 2001; Pal y Ireland, 2009)—o a temáticas legitimatorias referidas al constitucionalismo global (Kjaer, 2009). No obstante, estas tendencias pueden definirse como "un conjunto relativamente estable de relaciones de naturaleza no jerárquica e interdependiente que permite conectarse a diversos actores, quienes comparten intereses respecto de una política, intercambian recursos para alcanzar esos intereses compartidos y reconocen que la cooperación es la manera óptima de lograr objetivos comunes" (Börzel, 1998, p. 254).

Joop Koppenjan, Kars y van der Voort (2009) han identificado con claridad el problema políticosociológico detrás de este modelo. Por una parte, los actores político-democráticos enfatizan la relación entre agentes principales y autoridades políticas, mientras que las decisiones relevantes de ejecución son tomadas en niveles descentralizados (privados, cuasiprivados) de gobernanza. Por otra parte, los actores descentralizados tienen la experticia y capacidad para llevar adelante sus objetivos (también Willke, 1995), pero les es difícil generar apoyo político para evitar intervenciones desde niveles superiores. En tal sentido, una policy network puede ser entendida como el acoplamiento de la verticalidad de la democracia representativa y la horizontalidad de las múltiples formas de gobernanza de actores privados que se sitúan fuera del dominio de relaciones de la democracia representativa. Para lograr este acoplamiento, Koppenjan, Kars y van der Voort (2009) proponen el desarrollo de un marco de condiciones (framework setting) que regule la relación entre participantes y los límites procedimentales de la acción conjunta. Al hacerlo, deben enfrentarse a tres tipos de problemas: la complejidad, la interdependencia y la dinámica de todo policy issue. El primero de ellos debe ser superado por medio de un constante diálogo entre los participantes; el segundo, mediante la consideración del marco de condiciones como un acoplamiento suelto (loose coupling) (Weick, 1976) que permita desviaciones ante posibles contingencias y, el tercero, por medio de una apertura al aprendizaje a partir de la propia dinámica de implementación de la política.

Aun cuando se trate de acoplamientos sueltos, las policy networks suponen elementos que incentivan su mantención. Uno de ellos es la dependencia mutua de recursos: fondos, legitimación, capacidades de ejecución, 
información y elementos político-institucionales (Park, Rethemeyer y Hatmaker, 2009); otros elementos altamente relevantes son los recursos socioestructurales, esto es, aquellos "patrones de comunicación e intercambio de recursos entre tres o más actores" (Hatmaker y Rethemayer, 2008, p. 430) cuya estabilidad depende de los rendimientos para quienes las sostienen. Por su parte, el capital social de los participantes también contribuiría a un mejor desempeño de la red y con ello a su continuidad (Sandström y Carlsson, 2008). Sin embargo, un aspecto central de su operación parece ser su funcionamiento sobre la base de la producción de bienes colaterales, es decir, bienes que el Estado quiere, pero no puede producir por carencia de recursos y experticia, y que los privados no producen por falta de garantías ante el surgimiento de incentivos para agentes oportunistas (free-riders) o de un marco de condiciones apropiado (Willke, 1996). Los bienes colaterales corresponden a una reformulación estructural de los bienes colectivos cuya no competitividad desincentiva su producción. En términos de policy networks, los bienes colaterales suponen dependencia mutua (público-privada) de recursos, un marco para su operación, patrones de comunicación e intercambio relativamente estabilizados, orientación a un policy issue, y un alto nivel de uso de conocimiento y capacidades ejecutivas. El caso de los sistemas de concesiones de infraestructura en Chile responde a estas características.

La carencia de recursos financieros, conocimientos especializados y capacidad de ejecución, junto con el déficit de infraestructura que había en Chile a inicios de los años noventa, fueron incentivos clave para el desarrollo de bienes colaterales en el sector de obras públicas. Hasta la aprobación de la Ley de Concesiones en 1996 (MOP, DS $\mathrm{N}^{\circ} 900$ ), el financiamiento y la ejecución eran estatales. Hacia fines de los años setenta se introdujo la subcontratación para la construcción y mantenimiento de obras públicas, pero el diseño y la administración siguieron centralizados (Engel, Fischer y Galetovic, 2001). Con el sistema de concesiones, a los agentes públicos les corresponde fundamentalmente un papel regulador; las otras funciones quedan en manos de agentes privados en las distintas normativas: autopistas, aeropuertos, sistema de aguas, cárceles, puertos, entre otros.

En los términos anteriormente expuestos se puede hablar aquí de una policy network en formación. Se reúnen agentes públicos y privados en torno de la producción de bienes colaterales bajo una normativa determinada, en la que se distribuyen costos y beneficios de manera autorregulada por un marco de tipo jurídico y deliberativo. Este marco está compuesto por los institutos jurídicos pertinentes (Ley de Concesiones, reglamento, convenio-mandato, bases de licitación, oferta técnica y Decreto de Adjudicación), los contratos tipo acuerdos de construcción-explotación-transferencia (вОт, por sus siglas en inglés) o de diseño-construcción-explotacióntransferencia (DВОт, por sus siglas en inglés) y las comisiones de conciliación y de arbitraje. Mientras en los dos primeros se establecen los procedimientos de adjudicación e implementación, las últimas se orientan a las controversias que surjan en fases de ejecución y operación (Figueroa, 2003). El espacio deliberativo que abren estas dos instancias es central para su constitución como policy network, en tanto otorgan reflexividad al marco propiamente jurídico ante condiciones cambiantes del entorno del contrato. Especialmente con la comisión de conciliación se estabilizan patrones de comunicación e intercambio entre los agentes frente a controversias producidas que, de no ser resueltas, son conocidas por la misma comisión ahora en forma de comisión arbitral. Ambas partes pueden recurrir a la comisión conciliadora: por incumplimiento del contrato debido a fuerza mayor o destrucción de obras, por ejemplo, en el caso del Estado; por modificación de servicios o tarifas, retrasos imputables al Estado o suspensión de la concesión, en el caso de los privados (Figueroa, 2009).

Un marco de esta naturaleza puede parecer poco flexible para un acoplamiento suelto de actores. Sin embargo, los márgenes de flexibilidad e incentivos a la formación de policy networks son altos:

i) Los contratos tipo вОТ-DВОT operan sobre la base de agentes privados en consorcio. No es una subcontratación directa por parte del Estado; los actores privados autodefinen a sus socios (partners) y sus roles.

ii) La comisión conciliadora la integran "profesionales universitarios", es decir, expertos designados por las partes, pero no necesariamente vinculados directamente a ellas.

iii) La propia comisión determina sus normas y procedimientos aplicables, mecanismos de prueba, reclamo y notificación.

iv) Cualquiera de las partes puede solicitar la constitución de la comisión de conciliación.

v) El concesionario puede optar por una comisión de arbitraje o por la Corte de Apelaciones de Santiago si en el plazo de 30 días no hay conciliación (DS MOP N 900 art. 36; también Mensaje Presidencial $\mathrm{N}^{\circ} 358-355,2007$, p. 14).

Estos elementos son característicos de policy networks. Primero, en estos proyectos de alta complejidad e 
inversión tecnológica el conocimiento está distribuido. No solo el Estado es consciente de sus limitaciones en este aspecto, sino también la formación de consorcios y de un panel de expertos (comisión conciliadora) evidencia la distribución de conocimiento entre agentes privados. Se podría señalar que no solo el capital social contribuye al desempeño de la red, sino también — principalmente-el capital cultural bajo la forma de conocimiento (Willke, 2007). Segundo, la autoconstitución de la comisión en cuanto a normas y procedimientos, y la flexibilidad de vías de solución de controversias que abre revelan la operación sobre el principio de "autonomía de la voluntad" de las partes (Mereminskaya y Mascareño, 2005), lo que indica un ejercicio reflexivo de autovinculación y autolimitación de los actores involucrados. Tercero, lo anterior implica que la discutida renegociación de contratos (Guasch, Laffont y Straub, 2007; Rivera, 2008; Engel y otros, 2009) es inmanente a los del tipo вот у а la naturaleza flexible de una policy network. Una indeterminable cantidad de riesgos fluyen en ellos y hacen de la disyuntiva entre proceder y no proceder (go/no-go) un problema de modelación de riesgos y, por tanto, de manejo de conocimiento (Ock y otros, 2005). La proliferación de convenios complementarios es la consecuencia de este manejo de riesgos, y el proyecto de modificación de la Ley de Concesiones fue su respuesta institucionalizada.

En esta modificación se advierten ciertas limitaciones para la consolidación de una policy network. Se propone la creación del Consejo de Concesiones como una instancia "consultiva" de orientación de la política $\mathrm{y}$, por consiguiente, de formulación de metadecisiones que reúne tanto a representantes de distintas agencias públicas bajo la forma de una coordinación interministerial como a "especialistas" independientes del sector público y concesionarias, pero nombrados centralizadamente por el Presidente de la República (Mensaje Presidencial, $\mathrm{N}^{\mathrm{o}} 358-355$, art. 1, $\mathrm{n}^{\mathrm{o}} 2$ ).

Fundamental en toda policy network es la multiplicación de observaciones, particularmente en cuestiones de obras públicas con consecuencias para otros campos. Así también, la incorporación de conocimiento es determinante para un mejor desempeño de la red. Sin embargo, la no inclusión de actores privados en ella verticaliza las relaciones de gobernanza y constituye una limitación al carácter descentralizado de una policy network. No se trata solo de la exclusión de representantes de las concesionarias, sino principalmente de los públicos afectados por las obras. La no incorporación de posibles afectados aumenta el riesgo de las decisiones, pues ellas son tomadas sin consideración explícita de los públicos (Luhmann, 2006). Esto conduce a reacciones ex post y, finalmente, a mayores costos de transacción.

La introducción de una Superintendencia de Obras Públicas, orientada a la fiscalización de niveles de servicio (Mensaje Presidencial, No 1.194-356, art. 2), pretende ser una respuesta a estos riesgos; sin embargo, las policy networks requieren espacios deliberativos y no solo regulativos. Tampoco contribuye mayormente el cambio de árbitro arbitrador a árbitro mixto (art. 36). El primero tiene un espacio de deliberación más amplio: puede apartarse de la ley recurriendo a normas interpretativas, principios generales de derecho, legislación comparada y equidad; el segundo debe atenerse a derecho en su fallo sobre el fondo del asunto (Figueroa, 2003). Con esto se excluye la consideración del entorno de red en las decisiones arbitrales, como también la evaluación de la experiencia internacional en estas materias globales, cuestión vital para la consolidación de policy networks heterárquicas.

En síntesis, si el dilema central de las policy networks es el enfrentamiento de la verticalidad de las instituciones democrático-representativas con la horizontalidad de las formas privadas de gobernanza (Koppenjan, Kars y van der Voort, 2009), las modificaciones propuestas parecen enfatizar las líneas de verticalidad antes que las de horizontalidad. Con ello tienden a reducirse los grados de autonomía de los actores e incluso sus opciones de participación en una policy network, con los consecuentes desincentivos a formar parte de ella: si una vez abiertos la legislación tiende a reducir los espacios de libertad, las policy networks pueden adquirir un carácter ritualista y perder efectividad como mecanismo descentralizado de coordinación. 


\section{IV}

\section{El modelo de sistemas de deliberación: el caso de las comisiones asesoras presidenciales}

Formas de interrelación entre actores, como el Consejo de Concesiones, responden a lo que aquí se denomina sistemas de deliberación (Beyme, 1983; Scharpf, 1993; Willke, 1995; Parkinson, 2006; Whitman, 2007; Dryzek, 2009). Se trata de comisiones, consejos, mesas redondas de diálogo, foros, paneles de discusión y de expertos que reúnen a diversos actores corporativos, técnicos o agentes representativos de instancias diversas que pueden verse afectados por decisiones de política. Los sistemas de deliberación son un cuerpo constitutivo de policy networks, pero pueden formarse también con independencia de ellas con fines de discusión de políticas.

Teóricamente, con los sistemas de deliberación se aspira a la combinación de múltiples observaciones en torno de un tópico de interés para los actores convocados. Son un mecanismo articulador descentralizado que presupone altos grados de autonomía en las instancias participantes en cuanto a intereses sustantivos y procedimientos para alcanzarlos. La deliberación constituye precisamente un reconocimiento de esta heterarquía de actores y de la necesidad de su mutua referencia e interpelación para lograr - por medio de un acto de balance-compromisos en materias que les competen, sin que ello implique necesariamente un cambio en las preferencias, aunque sí la posibilidad de referir políticamente a una mayor legitimidad (Habermas, 2000; Ulbert y Risse, 2005). Para producir este efecto, un sistema político debe poseer - más allá de representación parlamentaria - mecanismos de deliberación procedimentales (no coercitivos, basados en principios), inclusivos (abiertos a múltiples intereses) y con consecuencias (esto es, con repercusión en decisiones de política) (Dryzek, 2009). La capacidad de producir consecuencias puede derivar de una participación directa en la formación de políticas (strong public space) o de influencia indirecta de foros informales en personas responsables de adoptar decisiones (weak public space) (Janssen y Kies, 2005). En cualquiera de los casos, elementos de un sistema deliberativo son un espacio público con mínimas limitaciones de participación de actores institucionales, o un espacio (privado) empoderado con vías de comunicación con el primero, más mecanismos de rendición de cuentas (accountability) e influencia en las decisiones (Dryzek, 2009, pp. 1.3851.386). En tanto, condiciones de emergencia y operación de sistemas deliberativos son: i) reconocimiento de una situación problemática para actores público-privados; ii) tolerancia a la ambivalencia y desequilibrios iniciales que solo se resuelven mediatamente con una interacción calculada a largo plazo; iii) desarrollo de confianza derivada de interacción a largo plazo, aceptación de sorpresas y nuevas opciones, y iv) capacidad reflexiva para incorporar perspectivas de otros a una ganancia combinatoria futura (óptimo de Kaldor), limitando la maximización presente de beneficios (óptimo de Pareto) (Willke, 1995 y 1997; Barabas, 2004).

El mecanismo de coordinación que prima en estas situaciones es el de una orientación contextual opuesta a una pura intervención jerárquico-autoritativa y a una autarquía de actores independiente de toda consideración del contexto (Willke, 1997). La orientación contextual en sistemas de deliberación establece condicionamientos y opciones por medio de los cuales los actores, sin desconocer la autonomía propia, pueden orientarse mutuamente combinando opciones en un marco flexible de posibilidades. Es decir, vinculan principios de coherencia y autonomía. En el mejor de los casos, sus resultados son: i) sentimientos de eficacia interna, un aspecto de una ciudadanía autónoma; ii) percepciones de mayor capacidad de respuesta gubernamental, como parte de la legitimidad política; iii) participación política, fundamental para una buena representación, y iv) compromiso cívico, identidad comunitaria y vecindad, que contribuyen a una comunidad democrática (Searing y otros, 2007, p. 612).

Sin embargo, los sistemas de deliberación no deben ser considerados una solución infalible a problemas de representatividad; ellos enfrentan diversas limitaciones. Primero, la reflexividad y confianza en otros actores se forman en el proceso. Para actores autónomos no es obvio limitar beneficios presentes en función de ganancias futuras. Especialmente si hay un problema que implica costos actuales distintos para cada uno, la tolerancia a la ambivalencia y los desequilibrios iniciales dependerá de 
la confianza de los actores en el procedimiento mismo y, por tanto, de la propia historia previa de resultados con sistemas de deliberación. Segundo, lo anterior conduce también a la figura de un "tercero": un actor imparcial dentro del sistema de deliberación o una fuente externa de autoridad. En sistemas de deliberación orientados a políticas, esta posición la asume una instancia gubernamental convocante, lo que restituye centralización por medio de atribuciones ideológicas y genera incentivos al agente imparcial y, por parte de los actores, se puede recurrir siempre a una idea determinada de "bien común" como fuente externa para sustentar intereses propios (Luhmann, 2005). Tercero, puesto que en ellos se combina una lógica de argumentación con una de negociación, no es posible esperar invariablemente consenso de los sistemas de deliberación. En ellos operan conjuntamente elementos de argumentación, persuasión y negociación que se interpenetran en la acción comunicativa (Ulbert y Risse, 2005) y que constituyen el paralelismo de metas (compromisos sin consenso) como un resultado más probable (Willke, 1996 y 2002).

El mecanismo de sistemas de deliberación ha sido empleado en Chile desde 1990 en adelante, sobre todo bajo la forma de comisiones asesoras y mesas de diálogo (Fuentes, 2006); estas últimas sobre todo en materias de derechos humanos. Según Carolina Aguilera (2007a, 2007b y 2009), fue distintivo del gobierno de Michelle Bachelet su implementación en directa relación con una semántica de "participación ciudadana" en la formulación de ideas de política. Para la autora, las comisiones del período Bachelet se distinguen entre aquellas que apuntan a una democracia participativa y las que se orientan a la eficiencia de políticas públicas. En la intención explícita del gobierno estos criterios se combinaban con tres objetivos: promoción de diálogo entre actores, formación de un espacio de participación y formulación de recomendaciones de política (Aguilera, 2009). La evaluación de la autora es matizada. Por una parte, se habría caído en una relación vertical al procesar gubernamentalmente a posteriori las propuestas de las comisiones y al incorporar solo expertos y no actores en algunas de ellas, aunque sí mecanismos de consulta (Aguilera, 2007b; Moulian, 2006); por otra, se reconoce que muchos expertos son también actores sociopolíticos y que las comisiones permitieron, al menos, acercar posiciones, establecer acuerdos mínimos y legitimar recomendaciones de política.

Su conclusión general es que: i) se constituyeron efectivamente espacios de diálogo y propuestas de política; ii) estos tuvieron un efecto limitado en cuanto a una amplia representación de intereses; iii) se incluyeron actores sociales en casos de protestas (como la comisión de calidad de la educación ante protestas estudiantiles, o la comisión de trabajo y equidad ante protestas de subcontratistas, entre otras); iv) permitieron un cambio de estilo político, pero en general siguieron vinculados a actores relevantes, y v) fue la discusión política en el Congreso la que en definitiva definió los proyectos de ley de políticas públicas.

La evaluación de Aguilera es interesante porque en ella se reflejan las posibilidades y limitaciones de los sistemas de deliberación en el caso chileno. Estos son un mecanismo probado para la vinculación de actores con distintos intereses y procedimientos de operación en torno de un tema específico que los afecta; asimismo, su incorporación es un reconocimiento de su autonomía. Por otra parte, la culminación de los procesos en informes revela que se lograron desarrollar niveles importantes de reflexividad. Y el hecho de que no logren consenso (como en el caso de la educación, en que estudiantes y profesores se marginaron del informe) indica que con lógicas de negociación es más probable que se pueda esperar una orientación hacia el paralelismo de metas. De igual modo, la tendencia a la experticia en sistemas de deliberación no puede entenderse por sí misma como tecnocratización; más bien revela que la complejidad y especialización de los problemas que Chile enfrenta en la actualidad exigen visiones metapolíticas, con intenso uso de conocimiento especializado e interdisciplinario, así como de experiencias internacionales comparadas.

Sin embargo, la ausencia de actores sociales relevantes supone en algunos casos (comisiones de previsión, infancia, educación superior, probidad) una importante limitación para alcanzar mayores niveles de reflexividad en los sistemas de deliberación, contrastando el conocimiento experto con el de los afectados. Como en el caso de las policy networks, esto aumenta los niveles de riesgo de los públicos a quienes eventualmente llegan las consecuencias de las decisiones tomadas, pues su experiencia directa no es incorporada. Sea debido a la complejidad logística de incluir a los afectados o al escaso desarrollo de actores corporativos en determinados campos en Chile, la exclusión de ellos en determinados sistemas de deliberación limita su efecto como mecanismo descentralizado de coordinación, tiende a tecnocratizarlo y a reducir la pluralidad de perspectivas respecto de un policy issue.

No obstante, las posibilidades y limitaciones de la experiencia con sistemas de deliberación en Chile son intrínsecas a este modelo. Es decir, pueden contribuir mediante el conocimiento experto y el de afectados a evaluaciones y recomendaciones de política, pero no 
están destinadas a sustituir a otros mecanismos democrático-deliberativos como discusiones parlamentarias, interministeriales y una esfera pública clásica, mediática o cotidiana, ninguno de los cuales, por lo demás, pasa los tests democráticos más exigentes (Johnston y Searing, 2005; Ulbert y Risse, 2005). En otros términos, los problemas de verticalización de sistemas deliberativos no provienen de que sus propuestas puedan ser discutidas en otros espacios democráticos, sino —en el caso chileno- de la centralización en la definición de sus miembros, de la exclusión de afectados no organizados o de la incompetencia del tercero imparcial para orientar hacia el equilibrio de las asimetrías emergentes durante los procesos de negociación, como también resalta en el análisis empírico de Aguilera (2009).

Los riesgos de verticalización también están presentes en los sistemas de deliberación. Sin embargo, por tratarse de mecanismos de coordinación de sistemas y actores con alta autonomía normativa y procedimental, el proceso de balance que se intenta siempre estará sometido a asimetrías y confrontaciones, más aún en sus fases iniciales de desarrollo, como es el caso chileno.

$\mathrm{V}$

\section{El modelo de derecho reflexivo para el caso del arbitraje en materias comerciales}

Una interesante forma de enfrentar estas asimetrías y generar paralelamente mecanismos de coordinación social heterárquicos es por medio de lo que se ha denominado derecho reflexivo. Para Gunther Teubner (1993), este consiste en la implementación de una política de opciones según la cual el derecho, abandonando pretensiones omnicomprensivas, produciría una regulación opcional que los interesados pueden aceptar o no. En este marco, el derecho reflexivo es vinculante si los afectados deciden vincularse. Subyace la premisa de que en un contexto de alta autonomía de actores y sistemas el derecho no puede obligar, integrar o dirigir conductas, pero sí ofrecerse como una opción de manejo de conflictos entre actores privados. El objetivo es incrementar la efectividad del derecho ofreciendo a los actores la posibilidad de sentirse motivados a operar con él.

El surgimiento de diversos regímenes jurídicos supranacionales de carácter privado es prueba de ello: lex mercatoria, lex sportiva, lex digitalis, lex financiaria, lex constructionis (Fischer-Lescano y Teubner, 2006 y 2007). En estos se desarrollan formas sustantivas y procedimentales de autorregulación, que en ocasiones colisionan y en otras se acoplan a los sistemas jurídicos nacionales (Mascareño, 2006a y 2006b). Característica común es la formación de paneles de decisión supranacionales para el tratamiento de conflictos. El arbitraje comercial internacional en el régimen de lex mercatoria es uno de ellos. Se trata de un mecanismo de resolución de controversias contractuales entre privados de distinta nacionalidad, aunque también opera para conflictos entre inversionistas extranjeros y Estado. Sus características centrales son:

i) La neutralidad del tribunal en tanto este no forma parte de ninguna estructura nacional.

ii) Su eficiencia en términos temporales al generar procedimientos "a medida" y carecer de instancias superiores de revisión de laudos.

iii) Su flexibilidad, derivada del principio de "autonomía de la voluntad" de las partes para definir autónomamente árbitros, lugar e idioma de arbitraje, plazos, pruebas aceptables y ley sustantiva aplicable.

iv) Su uso intensivo de conocimiento en tanto los árbitros son seleccionados por su especialización y experiencia en los temas de la controversia.

v) La opcionalidad regulatoria que ofrece a las partes mediante cláusulas en contratos y tratados (Buchanan, 1988; Guzmán, 2000; Mereminskaya, 2005).

Si bien el arbitraje comercial tiene un largo desarrollo, la intensificación de las relaciones comerciales y la consecuente proliferación de contratos privados y tratados entre Estados y regiones de la sociedad mundial en las últimas décadas han introducido novedosas consecuencias que pueden ser analizadas en términos de política pública. El incentivo de la actividad comercial y la atracción de inversiones no son solo materia privada. Las consecuencias en bienestar son también de interés público (empleo, conocimiento, infraestructura). Así como una desfavorable estructura de impuestos puede conducir a los inversores a seleccionar opciones externas (outside options) (Agostini y Jalile, 2009), también un 
régimen jurídico doméstico rígido puede desincentivar la actividad comercial y la inversión extranjera (Mereminskaya, 2004). El derecho mismo se transforma entonces en un bien público (Casella y Feinstein, 2002) cuya flexibilidad puede favorecer y su localismo puede limitar las relaciones económicas entre privados de distinta nacionalidad y entre ellos y los Estados.

Como forma de derecho reflexivo, el arbitraje es un mecanismo de alta adaptabilidad a las condiciones y diversas controversias de actores económicos autónomos. Esto lo provee de legitimidad entre sus usuarios, así como de una mayor reducción de incertidumbre, y construye un ambiente favorable para el comercio y la inversión (Mereminskaya, 2005). Incentivar su uso, por tanto, no puede estar ajeno a los objetivos de política.

Desde la ratificación de la Convención sobre el reconocimiento y ejecución de las sentencias arbitrales extranjeras (1958), la Convención de Panamá (1976) y la de Washington (1965), que establece el Centro Internacional de Arreglo de Diferencias Relativas a Inversiones (CIADI), pero especialmente desde la promulgación de la Ley sobre Arbitraje Comercial Internacional No 19.971 en 2004, Chile lo ha entendido de este modo. Esta ley es adoptada íntegramente a partir de la denominada Ley Modelo, elaborada por la Comisión de las Naciones Unidas para el Derecho Mercantil Internacional (CNUDMI) y recomendada a los Estados-miembro en 1985 (CNUDMI, 1985). Su objetivo es habilitar el arbitraje internacional con independencia de los tribunales nacionales, como explícitamente lo indica su artículo 50: "En los asuntos que se rijan por la presente Ley, no intervendrá ningún tribunal salvo en los casos en que esta Ley así lo disponga". El arbitraje tiene en Chile una larga tradición como arbitraje doméstico. En tanto mecanismo alternativo, ha parecido compatible con estructuras estamentales de reciprocidad altamente integradas (Mereminskaya, 2006; Xiao y Huo, 2005). En la actualidad, esto puede predisponer favorablemente a los actores hacia él. Sin embargo, la restricción jurisdiccional que establece la Ley 19.971 para tribunales domésticos conduce necesariamente a una fase de adaptación de estos a criterios supranacionales con consecuencias para el espacio económico y político.

Uno de los elementos más relevantes de esta fase de adaptación es la construcción por tribunales nacionales de una noción de orden público (normas no derogables que establecen límites contractuales) adecuada a criterios supranacionales. Frente a la revisión de laudos por nulidad en la Corte de Apelaciones o el reconocimiento de sentencias arbitrales extranjeras por la Corte Suprema, no es posible la aplicación mecánica de la noción doméstica de orden público, siempre más restrictiva que la internacional, la supranacional o la implícita en la lex fori (Buchanan, 1988). En el régimen arbitral internacional la única norma no derogable es la "buena fe" (UNIDRoIt, 2004, art 1.7; Mereminskaya, 2003; Mereminskaya y Mascareño, 2005).

En el ámbito doméstico, en cambio, incluso el art. 16 del Código Civil (aplicación de leyes chilenas a bienes situados en el país) ha sido considerado norma de orden público (Mereminskaya, 2006). La aplicación de este criterio constituye un obstáculo para el reconocimiento en Chile de sentencias arbitrales extranjeras, lo que desestabiliza, a nivel de la práctica judicial, el orden institucional política y legislativamente construido en que se opta por favorecer el acoplamiento jurídico con un orden transnacional y, en definitiva, se promueven relaciones comerciales internacionales e inversión extranjera. Frente a esta limitación anclada en la doctrina jurídica chilena, parece ser necesaria, en términos de política pública, la orientación de los cuerpos judiciales domésticos con respecto a las modalidades de aplicación de las Convenciones señaladas y las implicancias de la Ley 19.971.

Como en los casos anteriores (policy networks y sistemas de deliberación), la responsabilidad es tanto privada como pública. Por ello, la formación de policy networks en este campo puede ser de gran utilidad para una mayor coordinación entre privados y Estado. En una dinámica tal, a los primeros correspondería la coordinación y eficiente administración del arbitraje internacional en su dimensión procedimental, mientras que al Estado correspondería la supervisión y correcto funcionamiento de su institucionalidad jurídica en relación con criterios inter y supranacionales. Esto es cada vez más necesario ante la firma de múltiples tratados bilaterales en la última década que contienen cláusula de arbitraje (Direcon, 2009). A continuación se revisan consecutivamente estas dos dimensiones

Primero, en el ámbito privado, el arbitraje comercial internacional puede ser ad hoc (organizado por las partes) o institucional (en centros de arbitraje). Con el primero se evita el costo monetario del segundo, pero sus costos de transacción pueden ser demasiado altos, pues exige de las partes la planificación del arbitraje previo al conflicto. Esto incentiva el uso del arbitraje institucional. Siendo así, es esencial la estructuración procedimental eficiente del proceso arbitral y la claridad y simplicidad de la cláusula arbitral (Millet, 2006), así como la experticia de los administradores y la estabilidad de las condiciones organizacionales en que se desenvuelven; esto incluye: actualización académica de 
sus miembros, presencia en encuentros de la comunidad internacional de arbitraje, capacidad de convocarla a la sede institucional y adecuación organizacional a estándares de calidad internacionales.

Desde 1992 existe en Chile el Centro de Arbitraje y Mediación de Santiago (CAM-Santiago) de la Cámara de Comercio de Santiago, con alta eficiencia en el ámbito nacional y ahora abierto al arbitraje internacional. Como este, otros centros en América Latina han adquirido relevancia (en México, el Perú y el Brasil). Dados los procesos de liberalización económica en América Latina y la alta variabilidad regional y nacional, no parece recomendable la formación de un centro de arbitraje para la región, como sí puede serlo en el caso de Asia (Koh, 2000). Sin embargo, la informalidad institucional y el particularismo normativo en la región siguen siendo una limitación procedimental (Mascareño, 2006b). Por ello, parece primordial para estos centros de arbitraje formar policy networks que les permitan intercambio de conocimiento y experiencias de administración arbitral, niveles procedimentales comparables y transparencia en sus operaciones. Esta interacción también posibilita en el futuro la especialización y diferenciación temática de manera cooperativa entre distintos centros de arbitraje, con una consecuente reducción de costos alternativos.

Segundo, la proliferación de tratados bilaterales de inversión a nivel de Estados requiere también una coordinación en forma de policy networks de las instituciones arbitrales con los encargados estatales de diseñarlas y supervisarlas. Varias limitaciones doctrinarias y procedimentales que tienden a concentrar la resolución de disputas en el ámbito jurídico nacional pueden ser tratadas de este modo, por ejemplo: i) la eliminación de la doctrina
Calvo (resolución de disputas en cortes nacionales); ii) la revisión de la cláusula de la nación más favorecida cuando los derechos en disputa no están estipulados en el tratado bilateral de inversión, y iii) la estabilización de la cláusula paraguas utilizada en aquellos casos en que es necesario compatibilizar diferendos entre tribunales del contrato y del tratado (Cremades, 2004). Finalmente, la formación de policy networks público-privadas supranacionales en este campo puede llevar a incrementar los niveles de transparencia, información y confianza en el arbitraje internacional, limitaciones actualmente presentes en Chile y otros países de la región dada la creación relativamente reciente del sistema (en Chile es posible desde 2004, con la Ley 19.971). Alternativas para lo anterior pueden ser criterios coordinados de publicación de laudos por instituciones arbitrales (un aspecto criticado del arbitraje internacional) (Lord, 2001; Vandenbergh, 2005), o procedimientos para controlar las consecuencias del uso de información privilegiada, de corrupción o de privilegios familiares y de amistad en el ámbito público, cuestiones comunes en América Latina que desincentivan la inversión y fomentan la impredecibilidad del sistema.

En síntesis, las dinámicas generadas por la reflexividad del derecho en Chile y América Latina fomentan un principio de autonomía en actores y sistemas, como lo muestra el caso del arbitraje internacional. El desarrollo de policy networks en este campo es relevante para contrarrestar las limitaciones doctrinarias de la apertura jurídica nacional a criterios supranacionales, incrementar la confianza en el modelo y coordinar el campo político-legislativo de los tratados con el de los órdenes jurídicos domésticos.

\section{VI}

\section{Aproximación evaluativa de estos}

\section{modelos a nivel latinoamericano}

La coordinación social constituye un modo adecuado de enfrentar, por medio de políticas públicas, la creciente complejización social de actores y sistemas en el actual contexto chileno, latinoamericano y mundial. La alta autonomía sustantiva y procedimental ganada por estos actores es reactiva frente a un modo verticalista de relación entre políticas y públicos. Paralelamente, la autonomía supone riesgos si no va acompañada de mecanismos reflexivos que permitan tener en cuenta las consecuencias de las propias acciones de sistemas y actores en el entorno en que ellos mismos se desenvuelven. Autonomía implica también coherencia por la vía de la reflexividad.

En los modelos de policy networks, sistemas de deliberación y derecho reflexivo se procura combinar esos dos principios y articular a actores y sistemas 
privados y públicos mediante negociaciones, mesas redondas, ejercicios de autolimitación de expectativas por expectativas externas, visiones orientadas a problemas, contratos opcionales y autovinculación, en estructuras de coordinación social relativamente estables. En tres casos chilenos se muestra la efectividad y también las limitaciones de estas estrategias para enfrentar contextos sociales complejos. Es indiscutible la autonomía ganada por diversos actores y sistemas en el contexto latinoamericano en las últimas décadas; sin embargo, también es indiscutible la supervivencia de tendencias Estado-céntricas, ya sea bajo la forma de un marcado presidencialismo, de populismo corporativista o de tendencias autoritativas en la matriz sociopolítica de América Latina (Garretón, 2000).

Por otra parte, las condiciones de desigualdad social también atentan contra la formación de actores autónomos y abren espacios para la construcción de identidades comunitarias fuertes que se resisten a la deliberación o para resolver conflictos por la vía de la coerción o la corrupción (Mascareño, 2009). Bajo estas condiciones, el desarrollo de estrategias heterárquicas de coordinación es difícil de sostener. Mecanismos como los Consejos Comunales en la República Bolivariana de Venezuela apuntan a la consolidación ideológica del socialismo bolivariano antes que a la formación de mecanismos participativos de diseño y supervisión de políticas (Romero, 2007). Algo similar sucede con las asambleas constituyentes en la República Bolivariana de Venezuela, el Ecuador y el Estado Plurinacional de Bolivia: se aspira a legitimar una construcción política previa antes que la articulación de perspectivas. Asimismo, la elevada conflictividad armada en Colombia hace atractiva la selección de formas autoritativas de decisión. Por otra parte, el clientelismo en el modelo argentino se muestra reacio a la construcción de actores autónomos, y en el caso de Chile - como en el del Brasil con sus cámaras sectoriales de política industrial (Diniz, 1995) - se evidencia un relativo éxito en la construcción de estos mecanismos, pero la orientación a la concentración política de las decisiones finales y la exclusión de los potenciales afectados en algunos casos muestran sus actuales límites.

Cualquier tendencia Estado-céntrica limita la formación y efectividad de mecanismos descentralizados como las policy networks, los sistemas de deliberación y la política de opciones de un derecho reflexivo para controversias supranacionales. El presidencialismo concentra decisiones, el populismo las absorbe ante la incapacidad institucional de dar cuenta de las demandas y los modelos autoritativos las esconden. La pregunta en el caso latinoamericano es cuánto pueden resistir estas construcciones políticas verticales sin abrir una ventana ante la creciente complejidad social de la región. Los efectos de la globalización económica se expresan en diversos tratados bilaterales y multilaterales de comercio e inversión; varios agentes privados desarrollan sus estrategias de acción en términos regionales o globales más que nacionales; distintos actores demandan sus derechos con perspectiva transnacional antes que local, y su movilidad transregional (hacia Europa, América del Norte o Asia) es notoriamente mayor que en las décadas finales del siglo XX.

Con sus limitaciones actuales, se advierte en el caso chileno la incipiente formación de mecanismos de coordinación social mediante políticas públicas en el contexto latinoamericano. Esta parece ser una opción atendible y necesaria para el despliegue de tales políticas cuando recurrir al control estatal vertical se muestra inapropiado para enfrentar una creciente complejidad social. Hacerlo de este modo implica ventajas tanto para el Estado como para los actores privados. Sin perder la orientación general de prioridades temáticas, el primero se descarga en parte de la tarea de un diseño exhaustivo de sociedad y de la burocratización e inversión temporal que ello supone; los segundos pueden poner a disposición de ese diseño su conocimiento práctico y técnico, su experiencia como afectados por las consecuencias de política, y ganar con ello reconocimiento y autonomía de acción. Mecanismos de coordinación como las policy networks, los sistemas de deliberación y la política de opciones del derecho reflexivo son una atractiva alternativa en el contexto latinoamericano cuando las estructuras políticas tradicionales se ven presionadas por actores y sistemas que demandan, o ya ejercen, autonomía y pretensiones de participación y reconocimiento. Sin embargo, esas mismas estructuras constituyen límites para su realización, por lo que más cabe esperar una tensión que un cambio en este sentido. El despliegue de esa tensión mostrará su viabilidad futura en la región latinoamericana. 
Agostini, C. e I. Jalile (2009), "Efectos de los impuestos corporativos en la inversión extranjera en América Latina”, Latin American Research Review, vol. 44, No 2, Albuquerque, Latin American Studies Association.

Aguilera, C. (2009), "Un análisis de las comisiones asesoras presidenciales del gobierno de Michelle Bachelet", Documento de trabajo, Santiago de Chile, Facultad Latinoamericana de Ciencias Sociales (FLACSO).

(2007a), "Las comisiones de la Presidenta Bachelet", Diálogos de políticas públicas, $\mathrm{N}^{\circ} 1$, año 1 , Santiago de Chile, Facultad Latinoamericana de Ciencias Sociales (FLACSO).

(2007b), "Participación ciudadana en el gobierno de Bachelet: consejos asesores presidenciales", América Latina Hoy, vol. 46, Salamanca, Ediciones Universidad de Salamanca.

Arellano, J. (1985), "Social policies in Chile: an historical review", Journal of Latin American Studies, vol. 17, № 2, Cambridge, Cambridge University Press.

Barabas, J. (2004), "How deliberation affects policy options", American Political Science Review, vol. 98, No 4, Washington, D.C., American Political Science Association.

Bevir, M. y D. Richards (2009), "Decentring policy networks: a theoretical agenda", Public Administration, vol. 87, $\mathrm{N}^{\circ} 1$, Oxford, Blackwell Publishing.

Beyme, K. von (1994), Teoría política del siglo XX, Madrid, Alianza.

(1983), "Neo-corporatism: a new nut in an old shell?", International Political Science Review, vol. 4, No 2, Londres, Sage Publications.

Börzel, T. (1998), "Organizing Babylon - On the different conceptions of policy networks", Public Administration, vol. 76, $\mathrm{N}^{\mathrm{o}} 2$, Oxford, Blackwell Publishing.

Buchanan, M. (1988), "Public policy and international commercial arbitration", American Business Law Journal, vol. 26, № 3, Hoboken, John Wiley \& Sons.

Casella, A. y J. Feinstein (2002), "Public goods in trade: on the formation of markets and jurisdictions", International Economic Review, vol. 43, $\mathrm{N}^{\mathrm{o}}$ 2, Filadelfia, Universidad de Pensilvania/ Osaka University Institute of Social and Economic Research Association.

CNUdMI (Comisión de las Naciones Unidas para el Derecho Mercantil Internacional) (1985), "333 $3^{\text {rd }}$ Meeting", Yearbook of the United Nations Commission on International Trade Law, Washington, D.C.

Cremades, B. (2004), "Disputes arising out of foreign direct investment in Latin America: a new look at the Calvo Doctrine and other jurisdictional issues", Dispute Resolution Journal, Nueva York, American Arbitration Association, mayo-julio.

DeLeon, P. y D. Varda (2009), "Toward a theory of collaborative policy networks: identifying structural tendencies", Policy Studies Journal, vol. 37, N ${ }^{\circ} 1$, Hoboken, John Wiley \& Sons.

Diniz, E. (1995), "Reformas económicas y democracia en el Brasil de los años noventa: las cámaras sectoriales como foro de negociación”, Revista mexicana de sociología, vol. 57, № 4 , México, D.F., Universidad Nacional Autónoma de México.

Direcon (Dirección General de Relaciones Económicas Internacionales) (2009), "Acuerdos comerciales por país" [en línea] http:// rc.direcon.cl.

Domingues, J.M. (2008), Latin America and Contemporary Modernity, Londres, Routledge.

Dryzek, J. (2009), "Democratization as deliberative capacity building", Comparative Political Studies, vol. 42, № 11, Thousand Oaks, Sage.

Engel, E., R. Fischer y A. Galetovic (2001), "El programa chileno de concesiones de infraestructura: evaluación, experiencias y perspectivas", La transformación económica de Chile,
F. Larraín y R. Vergara (comps.), Santiago de Chile, Centro de Estudios Públicos.

Engel, E. y otros (2009), "Renegociación de concesiones en Chile", Estudios públicos, $\mathrm{N}^{\circ} 113$, Santiago de Chile, Centro de Estudios Públicos.

Figueroa, J. (2009), "El arbitraje en los contratos de concesión de obras públicas en Chile” [en línea] http://www.camsantiago. com/articulos_online_3.htm.

(2003), La resolución de controversias en la Ley Chilena de Concesiones de Obras Públicas, Santiago de Chile, Editorial Metropolitana.

Fischer-Lescano, A. y G. Teubner (2007), "Fragmentierung des Weltrechts: Vernetzung globaler Regimes statt etatistischer Rechtseinheit", Weltstaat und Weltstaatlichkeit. Beobachtungen globaler politischer Strukturbildung, M. Albert y R. Stichweh, Wiesbaden, VS-Verlag.

(2006), Regime-Kollisionen. Zur Fragmentierung des globalen Rechts, Frankfurt, Suhrkamp.

Fuentes, C. (2006), "Democracia en Chile: desafíos normativos y procedimentales", Desafíos democráticos, C. Fuentes, Santiago de Chile, Lom.

Garretón, M.A. (2000), Política y sociedad entre dos épocas, Rosario, HomoSapiens.

Granovetter, M. (1973), "The strength of weak ties", American Journal of Sociology, vol. 78, $\mathrm{N}^{\circ} 6$, Chicago, University of Chicago Press.

Guasch, J., J. Laffont y S. Straub (2007), “Concessions of infrastructure in Latin America: government-led renegotiation", Journal of Applied Econometrics, vol. 22, № 7, Hoboken, John Wiley \& Sons.

Guzmán, A. (2000), “Arbitrator liability: reconciling arbitration and mandatory rules", Duke Law Journal, vol. 49, № 5, Durham, Duke University School of Law.

Habermas, J. (2000), Facticidad y validez, Madrid, Editorial Trotta. (1990), Teoría de la acción comunicativa, Madrid, Taurus.

Hasenfeld, Y. (1972), "People processing organizations: an exchange approach", American Sociological Review, vol. 37, Washington, D.C., American Sociological Association.

Hatmaker, D. y K. Rethemeyer (2008), "Mobile trust, enacted relationships: social capital in a state-level policy network", International Public Management Journal, vol. 11, $\mathrm{N}^{\circ} 4$, Londres, Routledge.

Hayek, F. von (1986), "El orden de mercado o catalaxia", Lecturas de economía política, J. Huerta de Soto (comp.), Madrid, Unión Editorial.

Hecht-Oppenheim, L. (1993), Politics in Chile: Democracy, Authoritarianism and the Search for Development, Boulder, Westview Press.

Heydebrand, W. (2003), "Process rationality as legal governance", International Sociology vol. $18, \mathrm{~N}^{\mathrm{o}} 2$, Thousand Oaks, Sage.

Janssen, D. y R. Kies (2005), "Online forums and deliberative democracy”, Acta política, vol. 40, No 3, Nueva York, Palgrave Macmillan.

Johnston, P. y D. Searing (2005), "Studying 'everyday political talk' in the deliberative system", Acta política, vol. 40, No 3, Nueva York, Palgrave Macmillan.

Kjaer, P. (2009), "The under-complexity of democracy", Festschrift für Gunther Teubner, № 65, G.P. Callies y otros, Berlin, De Gruyter.

Koh, P. (2000), "Enhancing economic co-operation: a regional arbitration centre for Asean?", The International and Comparative Law Quarterly, vol. 49, $\mathrm{N}^{\circ}$ 2, Cambridge, Cambridge University Press. 
Koppenjan, J., M. Kars y H. van der Voort (2009), "Vertical politics in horizontal policy networks: framework setting as coupling arrangement", Policy Studies Journal, vol. 37, № 4, Hoboken, John Wiley \& Sons.

Kraft, M. y S. Furlong (2009), Public Policy: Politics, Analysis, and Alternatives, Washington, D.C., CQ Press.

Lechner, N. (1999), "El estado en el contexto de la modernidad", Reforma del estado y coordinación social, N. Lechner, R. Millán y D. Messner (comps.), México, D.F., Plaza y Valdés.

(1997), "Tres formas de coordinación social", Revista de la CEPAL, N 61 (LC/G.1955-P), Santiago de Chile, abril.

Lord, I. (2001), "The law: an engine for trade", Modern Law Review, vol. 64, No 3, Oxford, Blackwell Publishing.

Luhmann, N. (2007), La sociedad de la sociedad, México, D.F., Herder.

(2006), Sociología del riesgo, México, D.F., Universidad Iberoamericana.

(2005), "Staat und Politik. Zur Semantik der Selbstbeschreibung politischer Systeme", Soziologische Aufklärung 4, N. Luhmann (comp.), Wiesbaden, VS-Verlag.

Marsh, D. y R.A.W. Rhodes (1992), Policy Networks in British Government, Oxford, Clarendon Press.

Mascareño, A. (2009), "Acción y estructura en América Latina. De la matriz sociopolítica a la diferenciación funcional", Persona y sociedad, vol. $23, \mathrm{~N}^{\circ} 2$, Santiago de Chile, Universidad Alberto Hurtado.

(2006a), "Ethic of contingency beyond the praxis of reflexive law", Soziale Systeme, vol. 12, № 2 .

(2006b), "Regímenes jurídicos en la constitución de la sociedad mundial", Cuestiones de política criminal en los tiempos actuales, R. Carnevali (coord.), Santiago de Chile, Editorial Jurídica.

Mayntz, R. (1993), "Policy-Netzwerke und die Logik von Verhandlungssystemen", Politische Vierteljahresschrift, № 24 . (1992), "Modernisierung und die Logik von Verhandlungssystemen", Journal für Sozialforschung, № 32 .

Mensaje No 358-355 (2007), "Mensaje de la Presidenta de la República con el que inicia un proyecto de ley que modifica la Ley de Concesiones de Obras Públicas y otras normas que indica" [en línea] http://sil.congreso.cl/pags/index.html

Mensaje No 1194-356 (2008), "Mensaje de la Presidenta de la República con el que inicia un proyecto de ley que crea la Superintendencia de Obras Públicas" [en línea] http://sil. congreso.cl/pags/index.html.

Mereminskaya, E. (2006), "Arbitraje doméstico e internacional en Chile: en búsqueda de la armonía", Arbitraje y mediación en las Américas, J. Vargas y F. Gorjón, Santiago de Chile, Centro de Estudios de Justicia de las Américas (CEJA).

(2005), "Arbitraje comercial internacional", Análisis crítico del derecho internacional privado chileno, M. Ramírez, Santiago de Chile, Lexis Nexis.

(2004), "Los cambios en la jurisprudencia chilena ante los nuevos vínculos comerciales con Estados Unidos y Europa", Persona y sociedad, vol. 18, № 2, Santiago de Chile, Universidad Alberto Hurtado.

(2003), "Buena fe contractual en la jurisprudencia chilena e internacional", Revista de derecho de la Universidad Central de Chile, vol. 9, $\mathrm{N}^{\circ}$ 4, Santiago de Chile, Universidad Central de Chile.

Mereminskaya, E. y A. Mascareño (2005), "La desnacionalización del derecho y la formación de regímenes globales de gobierno", Sesquicentenario del Código Civil de Andrés Bello: pasado, presente y futuro de la codificación, M. Martinic (comp.), Santiago de Chile, Lexis Nexis.

Messner, D. (1999), "Del Estado céntrico a la 'sociedad de redes'. Nuevas exigencias a la coordinación social", Reforma del estado y coordinación social, N. Lechner, R. Millán y F. Valdés (comps.), México, D.F., Plaza y Valdés.
Millet, J. (2006), "Building a regional arbitration center: Chile's 2004 International Commercial Arbitration Law", http://www. camsantiago.com/articulos_online_2.htm.

MOP (Ministerio de Obras Públicas) (1996), Decreto Supremo Nº 900, Santiago de Chile.

Moulian, T. (2006), "El gobierno de Michelle Bachelet: las perspectivas de cambio", osaL - Observatorio Social de América Latina, año 6, $\mathrm{N}^{\circ} 19$, Buenos Aires, Consejo Latinoamericano de Ciencias Sociales.

Ock, J. y otros (2005), "Improving decision quality: a risk-based go/no-go decision for build-operate-transfer (ВОT) projects", Canadian Journal of Civil Engineering, vol. 32, № 3, Ottawa, NRC Research Press.

Pal, L. y D. Ireland (2009), "The public sector reform movement: mapping the global policy network", International Journal of Public Administration, vol. 32, № 8, Londres, Routledge.

Park, H., K. Rethemeyer y D. Hatmaker (2009), "The politics of connections: assessing the determinants of social structure in policy networks", The Academy of Management Proceedings, Birmingham, Academy of Management.

Parkinson, J. (2006), Deliberating in the Real World: Problems of Legitimacy in Deliberative Democracy, Oxford, Oxford University Press.

Peruzzotti, E. (1999), "Modernization and juridification in Latin America: a reassessment of the Latin American Developmental Path", Thesis Eleven, vol. 58, № 1, Thousand Oaks, Sage.

Rethemeyer, K. y D. Hatmaker (2008), "Network management reconsidered: an inquiry into management of network structures in public sector service provision", Journal of Public Administration Research and Theory, vol. 18, No 4, Oxford, Oxford University Press.

Rivera, E. (2008), "La regulación económica como complemento de las licitaciones en las concesiones de obras públicas", Revista de la CEPAL, No 95 (LC/G.2382-P), Santiago de Chile, agosto.

Romero, R. (2007), Los consejos comunales: más allá de la utopía, Maracaibo, Universidad del Zulia.

Sandström, A. y L. Carlsson (2008), "The performance of policy networks: the relation between network structure and network performance", Policy Studies Journal, vol. 36, № 4, Hoboken, John Wiley \& Sons.

Scharpf, F. (2001), "Notes toward a theory of multilevel governance in Europe", Scandinavian Political Studies, vol. 24, N $\mathrm{N}^{\mathrm{o}}$, Hoboken, John Wiley \& Sons.

(1993), "Positive und negative Koordination in Verhandlungssystemen”, Politische Vierteljahresschrift, $\mathrm{N}^{\circ} 24$.

Searing, D. y otros (2007), "Public discussion in the deliberative system: does It make better citizens?", British Journal of Political Science, vol. 37, No 4, Cambridge, Cambridge University Press.

Teubner, G. (1993), Law as an Autopoietic System, Cambridge, Blackwell Publishers.

Ulbert, C. y T. Risse (2005), "Deliberatively changing the discourse: what does make arguing effective?", Acta política, vol. 40, №3, Nueva York, Palgrave Macmillan.

UNIDROIT (Instituto Internacional para la Unificación del Derecho Privado) (2004), "Principles of international commercial contracts" [en línea] www.unidroit.org/english/principles/ contracts/main.htm.

Vandenbergh, M. (2005), "The private life of public law", Columbia Law Review, vol. 105, № 7, Nueva York, Columbia Law School.

Weick, K. (1976), "Educational organizations as loosely coupled systems", Administrative Science Quarterly, vol. 21, No 1 , Ithaca, The Johnson School at Cornell University.

Whetten, D. y H. Aldrich (1979), "Organization setsize and diversity: people-processing organizations and their environments", Administration and Society, vol. 11, No 3, Thousand Oaks, Sage. 
Whitman, J. (2007), "The challenge to deliberative systems of technological systems convergence", Innovation, vol. 20, No 4 , Londres, Routledge.

Willke, H. (2007), Smart Governance, Frankfurt, Campus. (2002), Dystopia, Frankfurt, Suhrkamp.

(1997), Supervision des Staates, Frankfurt, Suhrkamp.
(1996), Ironie des Staates, Frankfurt, Suhrkamp. (1995), Systemtheorie III: Steuerungstheorie, Stuttgart, Gustav Fischer.

Xiao, Y. y Z. Huo (2005), “Ordre public in China's private international law", The American Journal of Comparative Law, vol. 53, № 3 , Nueva York, American Society of Comparative Law. 